\section{Penicillinallergie bei Kindern oft nur ein Verdacht}

\author{
Der Verdacht auf eine Arzneimittel- \\ sensibilisierung im Kindesalter be- \\ stätigt sich in vielen Fällen nicht. \\ Doch trotz ausgeschlossener Allergie \\ verweigern so manche Eltern aus \\ Sorge vor einer allergischen Reaktion \\ eine Behandlung mit Penicillin.
}

U nerwünschte Arzneimittelreaktionen bei Kindern sind ein häufiges Problem in Klinik wie Praxis. Neue Daten hierzu präsentierte PD Dr. Hagen Ott vom Katholischen Kinderkrankenhaus Wilhelmstift Hamburg. Eine systematische Auswertung von 102 Studien zu dieser Problematik ergab, dass es bei bis zu 10,3\% aller beteiligten Kinder zu unerwünschten Arzneimittelreaktionen gekommen war, die eine Klinikeinweisung erforderlich gemacht hatten. Während Medikamentengaben im Krankenhaus traten bei bis zu 16,8\% der Fälle Nebenwirkungen auf. Bei ambulant behandelten Patienten betrug die Inzidenz unerwünschter Arzneimittelwirkungen bis zu $11 \%$. Antiinfektiva waren die Substanzgruppe mit den häufigsten unerwünschten Arzneimittelreaktionen [PLoS One 2012; 7: e24061].

Sehr häufig wird als Ursache einer unerwünschten Reaktion eine Arzneimittelallergie vermutet. Der Verdacht hält jedoch nur in den wenigsten Fällen einer allergologischen Diagnostik stand, berichtete Ott. So konnte in einer aktuellen Studie der Verdacht auf eine Arzneimittelsensibilisierung nur bei $10,6 \%$ von 658 untersuchten Kindern bestätigt werden [Clin Exp Allergy 2012; 42: 123-30].

Vor allem bei Exanthemen während der Einnahme von Betalaktam-Antibiotika wird eine „Penicillinallergie“ vermutet, ohne dass eine zuverlässige Testung dies bestätigt, sagte Ott. Bei 88 Kindern, die wegen urtikarieller Hauterscheinungen oder makulopapulöser Ex- antheme in einer Notaufnahme vorgestellt worden waren, war der Patchtest in keinem Fall, ein intradermaler Test lediglich in elf und ein Bluttest in zwei Fällen positiv. Eine orale Provokation bestätigte bei sechs Kindern eine BetalaktamAllergie. Die meisten Kinder wiesen mindestens eine positive Virusserologie auf. Darunter waren 54 Kinder, bei denen die Provokation negativ geblieben war. In vielen Fällen kann somit eine Virusinfektion ein vermeintliches „Penicillin-Exanthem“ erklären. Eine orale Provokation bringt Sicherheit, während Haut- und Bluttests nicht ausreichend zuverlässig sind [J Allergy Clin Immunol 2011; 127: 218-22].

Doch welche Konsequenzen hat eine ausgeschlossene Penicillinallergie in der Praxis? Eine Nachbeobachtung von 170 Fällen, in denen der Verdacht auf eine Penicillinallergie durch Haut- und Provokationstests ausgeschlossen werden konnte, ergab: Bei 130 (76\%) der Kinder war nach der Allergietestung eine erneute Antibiotika-Gabe erforderlich geworden. Bei 59 wurde Penicillin gegeben, doch 24 der Elternpaare hatten eine Penicillingabe verweigert, weil sie nach wie vor eine allergische Reaktion befürchteten [Allergy Asthma Proc 2012; 33: 1604]. Eine entsprechende Aufklärung nach Arzneimitteltestungen sei daher unerlässlich, betonte Ott. Angelika Bauer-Delto

Ott H. Diagnostik allergischer Arzneimittelreaktionen bei Kindern. Fortbildungssymposium "Arzneimittelallergie"

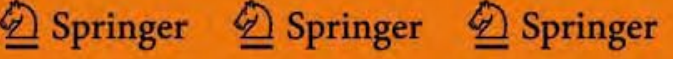
은 Springer Springer Springer Springer Springer Springer Springer Springe 은 Springer $\underline{\underline{2} \text { Springer }}$ Springer

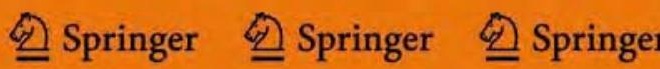
은 Springer Springer Q Springer $\underline{\text { Springer }}$ Springes Q Springer Springer

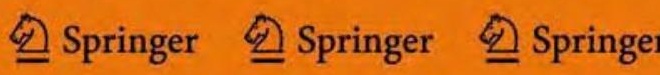
Springer Springer Springer Springer Springer Springe

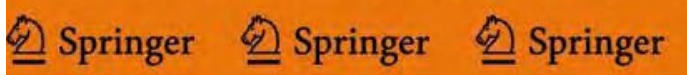
Q Springer Springer Springer

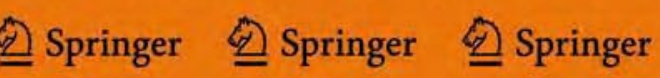
Q Springer Springer $\underline{\text { Springe }}$

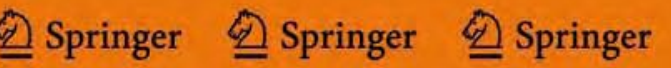
Springer Springer $\underline{\text { Springe }}$ 은 Springer $\underline{\text { Springer }}$ Springer Springer Springer 은 Springer Springer Springer Springer $\underline{\text { Springe }}$ 은 Springer Springer Springer $\underline{\text { Springer }}$ Springer Springer Springer Springer $\underline{\text { Springer }}$ Springe Springer $\underline{\text { Springer }}$ Springer Q Springer Springer $\underline{\text { Springe }}$

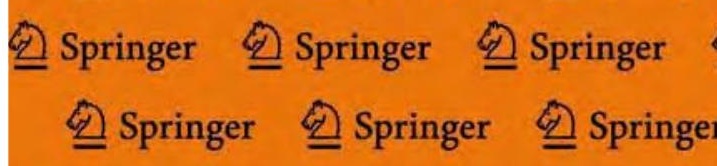
Q Springer Springer Springer Q Springer Springer Springe Springer 2 Springer 2 Springer 